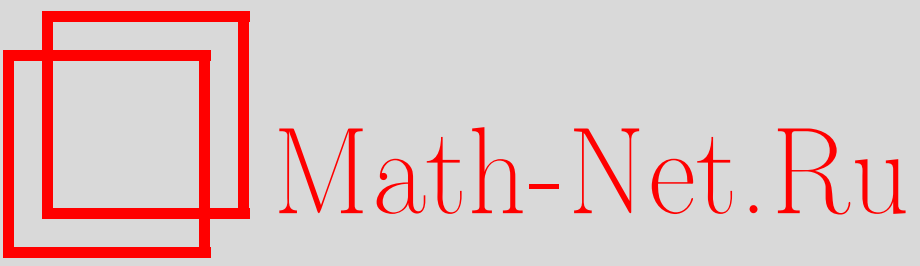

П. А. Кашицын, Многомерная модель с коррелированными наблюдениями, Теория вероятн. и ее примен., 2011, том 56, выпуск 3, 602-606

DOI: https://doi.org/10.4213/tvp4409

Использование Общероссийского математического портала Math-Net.Ru подразумевает, что вы прочитали и согласны с пользовательским соглашением

http://www.mathnet.ru/rus/agreement

Параметры загрузки:

IP : 3.80 .253 .173

26 апреля 2023 г., 06:06:22 
(C) $2011 \Gamma$

КАШИЦЫН П. А.*

\section{МНОГОМЕРНАЯ МОДЕЛЬ С КОРРЕЛИРОВАННЫМИ НАБЛЮДЕНИЯМИ}

Пусть мы наблюдаем $p$ количественных характеристик в $n$ временных точках. Таким образом, можно рассмотреть выборку из $n$ случайных $p$-мерных векторов $X_{1}, \ldots, X_{n}$. Относительно совместного распределения $p$-векторов делается предположение о том, что оно является нормальным и имеет ковариационную структуру, заданную в виде произведения Кронекера двух положительно определенных матриц.

В данной работе предлагаются методы для проверки линейных гипотез в многомерной модели с описанной структурой.

Ключевые слова и фразы: линейная гипотеза, коррелированные наблюдения, ковариационная структура, матричное произведение Кронекера, гауссовский многомерный анализ.

1. Введение. Рассмотрим выборку $p$-мерных случайных векторов $X_{1}, \ldots, X_{n}$. Из данных $p$-столбцов составим случайную $(p \times n)$-матрицу

$$
\mathbf{X}=\left\|X_{1}, \ldots, X_{n}\right\| .
$$

Введем понятие векторизации матрицы, которое сопоставляет каждой $(p \times n)$ матрице вектор длины $p n$, составленный из столбцов данной матрицы:

$$
\operatorname{vec}(\mathbf{X})=\operatorname{vec}\left(\left\|X_{1}, \ldots, X_{n}\right\|\right)=\left(X_{1}^{T}, \ldots, X_{n}^{T}\right)^{T} .
$$

Будем предполагать, что совместное распределение случайных векторов $X_{1}, \ldots, X_{n}$ является нормальным и подчинено закону

$$
\operatorname{vec}(\mathbf{X})=\left(X_{1}^{T}, \ldots, X_{n}^{T}\right)^{T} \sim N_{p \times n}(\operatorname{vec}(\mathbf{M}), \Psi \otimes \Sigma),
$$

где параметры модели $\mathbf{M}, \Psi$ и $\Sigma$ определены следующим образом:

$$
\begin{gathered}
\mathbf{M}=\left\|\mathbf{E} X_{1}, \ldots, \mathbf{E} X_{n}\right\|, \\
\operatorname{Cov}\left(X_{i}, X_{j}\right)=\psi_{i j} \Sigma, \quad i, j=1, \ldots, n .
\end{gathered}
$$

Предполагается, что матрицы $\Psi$ и $\Sigma$ являются положительно определенными, причем матрица $\Psi$ считается известной. В этом случае ковариационная матрица $\Lambda=$ $\Psi \otimes \Sigma$ случайного вектора $\operatorname{vec}(\mathbf{X})$, которая определяется через матричное произведение Кронекера:

$$
\Lambda=\Psi \otimes \Sigma=\left(\begin{array}{ccc}
\psi_{11} \Sigma & \ldots & \psi_{1 n} \Sigma \\
\vdots & \ddots & \vdots \\
\psi_{n 1} \Sigma & \ldots & \psi_{n n} \Sigma
\end{array}\right)
$$

также является положительно определенной матрицей.

* Московский государственный университет им. М.В.Ломоносова, механикоматематический факультет, кафедра теории вероятностей, Ленинские горы, 119991 Москва, Россия; e-mail: pavel.kash@gmail.com 
2. Обобщенная линейная модель с коррелированными наблюдениями. Рассмотрим левый алгебраический модуль $\mathbf{R}_{n}^{p}$, элементами которого являются $(p \times n)$ матрицы над кольцом $(p \times p)$-матриц. Таким образом, есть взаимно однозначное соответствие между элементами модуля и матрицами $\mathbf{X}$, которые построены в (1) по выборке $X_{1}, \ldots, X_{n}$. Введем аналог скалярного произведения в построенном модуле, а также определим ряд объектов, обобщающих понятия, введенные в работе [1].

О п р е д е л е н и е 1 . Скалярное произведение двух $(p \times n)$-матриц $\mathbf{X}$ и $\mathbf{Y}$ есть $(p \times p)$-матрица вида

$$
\langle\mathbf{X}, \mathbf{Y}\rangle_{Q}=\mathbf{X} Q \mathbf{Y}^{T}
$$

где $Q$ - положительно определенная $(n \times n)$-матрица; таким образом, билинейная форма, определяющая введенное скалярное произведение, имеет матрицу $Q$.

Далее, определим линейное преобразование как умножение элементов модуля на $(n \times n)$-матрицу справа.

О п р е д е л е н и е 2. Матрицей линейного преобразования назовем $(n \times n)$ матрицу $A$, которая действует по следующему правилу:

$$
\mathbf{X} \rightarrow \mathbf{X} A \text {. }
$$

О п р е д е л е н и е 3 . Ортогональным дополнением к подмодулю $\mathscr{L}$ назовем подмодуль $\mathscr{L}^{\perp}=\left\{\mathbf{Y} \mid \mathbf{Y} \in \mathbf{R}_{n}^{p},\langle\mathbf{Y}, \mathbf{X}\rangle_{Q}=0\right.$ для всякого $\left.\mathbf{X} \in \mathscr{L}\right\}$.

О п р е д е л е н и е 4 . Проекцией $(p \times n)$-матрицы $\mathbf{X}$ на подмодуль $\mathscr{L} \subset \mathbf{R}_{n}^{p}$ назовем $(p \times n)$-матрицу $\mathbf{Z}=\operatorname{proj}_{\mathscr{L}} \mathbf{X}$ такую, что

a) $\mathbf{Z} \in \mathscr{L}$

b) $\mathbf{X}-\mathbf{Z} \in \mathscr{L}^{\perp}$.

Теорема 1 (об ортогональном разложении). Пусть $\mathbf{X}=\left\|X_{1}, \ldots, X_{n}\right\|$ eсть $(p \times$ n)-матрица, удовлетворяющая (3), для которой $\operatorname{Cov}\left(X_{i}, X_{j}\right)=\psi_{i j} \Sigma, i, j=$ $1, \ldots, n$, причем матриџы $\Psi$ и $\Sigma$ являются положительно определенньми. Пусть $\mathscr{L}_{1}, \ldots, \mathscr{L}_{m}$ - попарно ортогональные подмодули введенного модуля $\mathbf{R}_{n}^{p}$ с матрицей скалярного произведения $I_{n}$, прямая сумма которых составляет $\mathbf{R}_{n}^{p}$ :

$$
\mathbf{R}_{n}^{p}=\mathscr{L}_{1} \oplus \cdots \oplus \mathscr{L}_{m} .
$$

Рассмотрим разложение $(p \times n)$-матриць $\mathbf{X} \Psi^{-1 / 2}$ на попарно ортогональные составляюиие:

$$
\mathbf{X} \Psi^{-1 / 2}=\operatorname{proj}_{\mathscr{L}_{1}}\left(\mathbf{X} \Psi^{-1 / 2}\right)+\cdots+\operatorname{proj}_{\mathscr{L}_{m}}\left(\mathbf{X} \Psi^{-1 / 2}\right) .
$$

Тогда:

а) случайнье $(p \times n)$-матриць $\operatorname{proj}_{\mathscr{L}_{i}}\left(\mathbf{X} \Psi^{-1 / 2}\right), i=1, \ldots, m$, независимьи, распределень нормально $и \mathbf{E} \operatorname{proj}_{\mathscr{L}_{i}}\left(\mathbf{X} \Psi^{-1 / 2}\right)=\operatorname{proj}_{\mathscr{L}_{i}} \mathbf{E}\left(\mathbf{X} \Psi^{-1 / 2}\right)$, где билинейная форма скалярного произведения, определяющего проекиию, имеет матричу $I_{n}$;

b) $\left\langle\operatorname{proj}_{\mathscr{L}_{i}}\left(\mathbf{X} \Psi^{-1 / 2}\right), \operatorname{proj}_{\mathscr{L}_{i}}\left(\mathbf{X} \Psi^{-1 / 2}\right)\right\rangle_{I_{n}}=W_{p}\left(\operatorname{dim} \mathscr{L}_{i}, \Sigma, \Delta_{i}\right)$, где случайная $(p \times$ p)-матрича $W_{p}\left(\operatorname{dim} \mathscr{L}_{i}, \Sigma, \Delta_{i}\right)$ распределена по Уишарту с параметром нецентральности

$$
\Delta_{i}=\left\langle\operatorname{proj}_{\mathscr{L}_{i}} \mathbf{E}\left(\mathbf{X} \Psi^{-1 / 2}\right), \operatorname{proj}_{\mathscr{L}_{i}} \mathbf{E}\left(\mathbf{X} \Psi^{-1 / 2}\right)\right\rangle_{I_{n}} .
$$

Доказательство теоремы сводится к теореме 5 об ортогональном разложении, установленной в [1], с помощью лемм, которые приведены ниже.

Лемма 1. Для положительно определенной симметричной матриць $A=$ $\left(a_{i j}\right)_{i, j=1}^{m}$ вьполнено следующее равенство:

$$
\left(A \otimes I_{p}\right)^{-1 / 2}=A^{-1 / 2} \otimes I_{p} .
$$

Лемма 2. Для симметричной матрицы $A=\left(a_{i j}\right)_{i, j=1}^{n}$ и матрицы $\mathbf{X}$, определенной в условии теоремы, выполнено следующее равенство:

$$
\operatorname{vec}(\mathbf{X} A)=\left(A \otimes I_{p}\right) \operatorname{vec}(\mathbf{X})
$$


Лемма 3. Для матрицы $\mathbf{X}$ из условия теоремы случайный вектор $\operatorname{vec}\left(\Sigma^{-1 / 2} \times\right.$ $\left.\mathbf{X} \Psi^{-1 / 2}\right)$ имеет распределение $N_{p \times n}\left(\operatorname{vec}\left(\Sigma^{-1 / 2} \mathbf{M} \Psi^{-1 / 2}\right), I_{n p}\right)$.

Следствие 1. Для матрицы $\mathbf{X}$ из условия теоремы случайный вектор $\operatorname{vec}\left(\mathbf{X} \Psi^{-1 / 2}\right)$ имеет распределение $N_{p \times n}\left(\operatorname{vec}\left(\mathbf{M} \Psi^{-1 / 2}\right), I_{n} \otimes \Sigma\right)$.

Представим скалярное произведение двух элементов модуля как композицию линейного преобразования с матрицей преобразования $\Psi^{-1 / 2}$ и стандартного евклидова скалярного произведения с единичной билинейной формой, определяющей скалярное произведение. А именно,

$$
\langle\mathbf{X}, \mathbf{Y}\rangle_{\Psi^{-1}}=\mathbf{X} \Psi^{-1} \mathbf{Y}^{T}=\left(\mathbf{X} \Psi^{-1 / 2}\right)\left(\mathbf{Y} \Psi^{-1 / 2}\right)^{T} .
$$

Таким образом, поскольку столбцы матрицы $\mathbf{X} \Psi^{-1 / 2}$ независимы, что вытекает из следствия 1 , то доказательство утверждения сводится к теореме 5 работы [1].

\section{3. Проверка линейных гипотез.}

О п р е д е л е н и е 5. Говорят, что матрица $\mathbf{X}$ из (1) со случайными элементами $X_{1}, \ldots, X_{n}$ следует линейной гауссовской модели с ковариационной структурой в виде произведения Кронекера, если

a) $\mathbf{E} \mathbf{X} \in \mathscr{U}$ для некоторого заданного подмодуля $\mathscr{U}$;

b) $\operatorname{vec}(\mathbf{X})$ имеет распределение $N_{p \times n}(\operatorname{vec}(\mathbf{M}), \Psi \otimes \Sigma)$, где параметры задаются формулами (4) и (5).

Линейной гипотезой в линейной модели назовем гипотезу

$$
H: \mathbf{E} \mathbf{X} \in \mathscr{U}_{1},
$$

где $\mathscr{U}_{1}$ - заданный подмодуль и $\mathscr{U}_{1} \subset \mathscr{U}$. Заметим, что данная гипотеза эквивалентна следующей:

$$
H: \mathbf{E}\left(\mathbf{X} \Psi^{-1 / 2}\right) \in \mathscr{U}_{1} \Psi^{-1 / 2} .
$$

Введем следующие обозначения:

$$
\mathscr{L}=\mathscr{U} \Psi^{-1 / 2}, \quad \mathscr{L}_{1}=\mathscr{U}_{1} \Psi^{-1 / 2} \quad \text { и } \quad \mathbf{Y}=\mathbf{X} \Psi^{-1 / 2},
$$

и разложим модуль $\mathscr{L}$ в ортогональную сумму подмодулей:

$$
\mathscr{L}=\mathscr{L}_{1} \oplus \mathscr{L}_{2}
$$

Рассмотрим разложение модуля $\mathbf{R}_{n}^{p}$ на три попарно ортогональных подмодуля:

$$
\mathbf{R}_{n}^{p}=\mathscr{L}_{1} \oplus \mathscr{L}_{2} \oplus \mathscr{L}^{\perp}
$$

В силу доказанной теоремы 1 случайные матрицы

$$
S_{1}:=\left\langle\operatorname{proj}_{\mathscr{L} \perp} \mathbf{Y}, \operatorname{proj}_{\mathscr{L} \perp} \mathbf{Y}\right\rangle_{I_{n}}, \quad S_{2}:=\left\langle\operatorname{proj}_{\mathscr{L}_{2}} \mathbf{Y}, \operatorname{proj}_{\mathscr{L}_{2}} \mathbf{Y}\right\rangle_{I_{n}}
$$

статистически независимы и распределены по Уишарту, где проекции и скалярный квадрат матриц определяются из теоремы 1.

Пусть $\operatorname{dim} \mathscr{L}=m, \operatorname{dim} \mathscr{L}_{1}=m_{1}$ и $\operatorname{dim} \mathscr{L}_{2}=m_{2}$, тогда

$$
S_{1} \sim W_{p}(n-m, \Sigma)
$$

Если гипотеза $H(9)$ верна, то

$$
S_{2} \sim W_{p}\left(m_{2}, \Sigma\right)
$$

При нарушении гипотезы $H$ распределение Уишарта статистики (11) становится нецентральным, приобретая параметр нецентральности

$$
\Delta=\left\langle\operatorname{proj}_{\mathscr{L}_{2}} \mathbf{E} \mathbf{Y}, \operatorname{proj}_{\mathscr{L}_{2}} \mathbf{E} \mathbf{Y}\right\rangle_{I_{n}}
$$


который тем больше, чем сильнее нарушена гипотеза $H$ в (9).

Рассмотрим матрицу $S_{2} S_{1}^{-1}$, представляющую собой аналог «эф»-отношения. Распределение данной матрицы не свободно от параметра и имеет вид

$$
\Sigma^{-1 / 2} W_{p}\left(m_{2}, I_{p}\right) W_{p}^{-1}\left(n-m, I_{p}\right) \Sigma^{-1 / 2} .
$$

Поскольку матрица (13) подобна матрице

$$
W_{p}\left(m_{2}, I_{p}\right) W_{p}^{-1}\left(n-m, I_{p}\right),
$$

распределение которой свободно, то в качестве критических статистик могут быть использованы собственные значения матрицы (13), которые совпадают с собственными значениями матрицы (14) и являются корнями характеристического уравнения относительно $\lambda$ :

$$
\operatorname{det}\left(W_{p}\left(m_{2}, I_{p}\right)-\lambda W_{p}\left(n-m, I_{p}\right)\right)=0 .
$$

4. Однофакторный дисперсионный анализ. Проиллюстрируем построенную линейную модель с зависимыми наблюдениями на примере однофакторного дисперсионного анализа. Рассмотрим $k$ выборок, где $k \geqslant 2$ и в $j$-й выборке содержится $n_{j}$ элементов. Обозначим элементы выборок через $X_{i j}$, где $i=1, \ldots, n_{j}$, $j=1, \ldots, k$. Соответственно $n_{1}, \ldots, n_{k}$ - объемы выборок, $N=\sum_{j=1}^{k} n_{j}$. Далее $X_{11}, \ldots, X_{n_{k} k}$ - это $p$-мерные векторы и $X_{\cdot_{1}}, \ldots, X_{\cdot k}$ - средние значения по столбцам. Пусть $\mathbf{X}=\left(X_{11}, \ldots, X_{n_{1} 1}, \ldots, X_{1 k}, \ldots, X_{n_{k} k}\right)$.

Статистическая модель: наблюдения из одной выборки независимы, а наблюдения из разных выборок зависимы, причем $X_{i j} \sim N_{p}\left(M_{j}, \Sigma\right), j=1, \ldots, k$, где векторы математических ожиданий $M_{1}, \ldots, M_{k}$ и матрица $\Sigma$ (общая для всех наблюдений) неизвестны. Будем считать, что $\Sigma$ невырождена. Пусть $\operatorname{Cov}\left(X_{i j}, X_{k l}\right)=\psi_{r s}$, где $r=i+\sum_{t=1}^{j-1} n_{t}, s=k+\sum_{t=1}^{l-1} n_{t}$, причем $\psi_{r s}=0$ при $j=l$, так как наблюдения из одной выборки независимы. Пусть также матрица $\Psi_{N}=\left(\psi_{r s}\right), r, s=1, \ldots, N$, положительно определена и считается известной и $N>p+k-1$.

Рассмотрим нулевую гипотезу о равенстве средних:

$$
H_{0}: M_{1}=\cdots=M_{k} .
$$

По-другому данную гипотезу можно записать следующим образом:

$$
H_{0}: \mathbf{E} \mathbf{X} \in \mathscr{U}_{1}=\left\{(\underbrace{M, \ldots, M}_{N}) \mid M \in \mathbf{R}^{p}\right\} .
$$

Тогда согласно введенной ранее терминологии имеем:

$$
\mathscr{U}=\left\{(\underbrace{M_{1}, \ldots, M_{1}}_{n_{1}}, \ldots, \underbrace{M_{k}, \ldots, M_{k}}_{n_{k}}) \mid M_{1}, \ldots, M_{k} \in \mathbf{R}^{p}\right\} .
$$

Выполним линейное преобразование модуля с матрицей преобразования $\Psi_{N}^{-1 / 2}$ :

$$
\mathbf{Y}=\mathbf{X} \Psi_{N}^{-1 / 2}, \quad \mathscr{L}=\mathscr{U} \Psi_{N}^{-1 / 2}, \quad \mathscr{L}_{1}=\mathscr{U}_{1} \Psi_{N}^{-1 / 2} .
$$

В результате преобразования по следствию 1 столбцы $\mathbf{Y}$ станут линейнонезависимыми. Оператор проекции вида $\left(A^{T}\left(A A^{T}\right)^{-1} A\right)$ на подмодуль размерности $r$, где $(r \times n)$-матрица $A$ составлена из порождающих модуль $n$-строк (см., например, [1]), после преобразования примет вид

$$
\Psi_{N}^{-1 / 2} A^{T}\left(A \Psi_{N}^{-1} A^{T}\right)^{-1} A \Psi_{N}^{-1 / 2} .
$$

Пусть $\mathscr{L}_{1} \oplus \mathscr{L}_{2}=\mathscr{L}$. Таким образом,

$$
S_{1}=\left\langle\operatorname{proj}_{\mathscr{L} \perp} \mathbf{Y}, \operatorname{proj}_{\mathscr{L} \perp} \mathbf{Y}\right\rangle_{I_{N}}, \quad S_{2}=\left\langle\operatorname{proj}_{\mathscr{L}_{2}} \mathbf{Y}, \operatorname{proj}_{\mathscr{L}_{2}} \mathbf{Y}\right\rangle_{I_{N}} .
$$


При сделанном предположении $N>p+k-1$ матрица $S_{1}$ невырождена с вероятностью 1 . В качество одной из критериальных статистик мы можем рассмотреть статистику Уилкса:

$$
\Lambda=\frac{\operatorname{det}\left(S_{1}\right)}{\operatorname{det}\left(S_{1}+S_{2}\right)} .
$$

Данная статистика распределена как произведение $p$ независимых случайных величин, следующих бета-распределениям с параметрами соответственно

$$
\left(\frac{N-k-p+1}{2}, \frac{k-1}{2}\right),\left(\frac{N-k-p+2}{2}, \frac{k-1}{2}\right), \ldots,\left(\frac{N-k}{2}, \frac{k-1}{2}\right) .
$$

Гипотезу $H_{0}$ отвергаем при малых значениях статистики $\Lambda$.

Автор выражает признательность своему научному руководителю профессору Ю. Н. Тюрину за постоянное внимание к работе.

\section{СПИСОК ЛИТЕРАТУРЫ}

1. Тюрин Ю.Н. Многомерный статистический анализ: геометрическая теория. Теория вероятн. и ее примен., 2010, т. 55, в. 1, с. 36-58.

2. Kashitsyn P. A. Multivariate model with a Kronecker product covariance structure: S. N. Roy method of estimation. - Math. Methods Statist., 2011, v. 20, № 1, p. 75-78.

3. Roy A., Khattree R. On implementation of a test for Kronecker product covariance structure for multivariate repeated measures data. - Statist. Methodol., 2005, v. 2, № 4, p. 297-306.

4. Srivastava M.S., von Rosen T., von Rosen D. Models with a Kronecker product covariance structure: estimation and testing. - Math. Methods Statist., 2008, v. 17, № 4 , p. $357-370$.

5. Srivastava M.S., Khatri C. G. An Introduction to Multivariate Statistics. New York: North-Holland, 1979, 350 p.

6. Votaw D.F. Testing compound symmetry in a normal multivariate distribution. Ann. Math. Statist., 1948, v. 19, p. 447-473.

Поступила в редакцию 26.IX.2010

Исправленный вариант

06.VII.2011

(c) 2011 г.

МАСТИХИН А.В.

\section{ФИНАЛЬНЫЕ ВЕРОЯТНОСТИ МАРКОВСКОГО ПРОЦЕССА ЭПИДЕМИИ БЕККЕРА}

Получены интегральные представления для производящих функций переходных и финальных вероятностей марковского процесса двойной эпидемии. Используется предложенная в работах [8], [10] свертка первой системы дифференциальных уравнений Колмогорова с помощью экспоненциальной производящей функции для ветвящихся процессов с взаимодействием. Установлена предельная теорема для числа финальных частиц в эпидемии.

* Московский государственный технический университет им. Н. Э. Баумана, кафедра высшей математики, 2-я Бауманская ул., 5, 105005 Москва, Россия; e-mail: mastikhin@yandex.ru 